\title{
Development and Characterization of Nanoparticles- Loaded Bio-composites for Biomedical Settings
}

\author{
Gustavo Hernandez-Vargas, Roberto Parra-Saldivar and Hafiz M.N. Iqbal* \\ Tecnologico de Monterrey, School of Engineering and Sciences, Monterrey, 64849, Mexico.
}

*Correspondence: hafiz.iqbal@tec.mx

(Received: October 02, 2020; accepted: October 20, 2020)

Citation: Hernandez-Vargas G, Parra-Saldivar R, Iqbal HMN. Development and Characterization of Nanoparticles-Loaded Biocomposites for Biomedical Settings. J Pure Appl Microbiol. 2020;14(4):2323-2337. doi: 10.22207/JPAM.14.4.11

C The Author(s) 2020. Open Access. This article is distributed under the terms of the Creative Commons Attribution 4.0 International License which permits unrestricted use, sharing, distribution, and reproduction in any medium, provided you give appropriate credit to the original author(s) and the source, provide a link to the Creative Commons license, and indicate if changes were made. 


\section{Abstract}

There is a dire need to engineer biologically robust constructs to meet the growing needs of $21^{\text {st }}$ century medical sector. The increasing (re)-emergence of human-health related pathogenic microbes has caused a havoc and serious challenge to health care services. In this context, herein, we report the development and characterization of various polymeric bio-composites with unique structural and functional attributes. For a said purpose, chitosan and graphene were used to engineer bio-composites, which were then functionalized by loading silver and platinum nanoparticles. A microwave-assisted approach was adopted to construct silver and platinum nanoparticles loaded graphene-based bio-composites. While, "one-pot" synthesis approach was used to engineer silver and platinum nanoparticles loaded chitosan-based bio-composites. As developed bio-composites were designated as GO-Ag-S1 to GO-Ag-S5 (silver nanoparticles loaded graphene-based bio-composites), GO-Pt-P1 to GO-Pt-P5 (platinum nanoparticles loaded graphene-based bio-composites), $\mathrm{CHI}-\mathrm{Ag}-\mathrm{S} 1$ to $\mathrm{CHI}-\mathrm{Ag}-\mathrm{S} 5$ (silver nanoparticles loaded chitosan-based bio-composites), and CHI-Pt-P1 to CHI-Pt-P5 (platinum nanoparticles loaded chitosan-based bio-composites). Finally, the nanoparticles loaded bio-composites of graphene and chitosan were subjected to characterization via UV-Visible spectrophotometric analysis, percent loading efficiency (\%LE) analysis, Fourier-transform infrared (FTIR) spectroscopy, mechanical measurements, and antibacterial attributes. The UV-Visible spectrophotometric analysis revealed characteristic peaks appeared at the $\lambda_{\max } 420 \mathrm{~nm}$ and $266 \mathrm{~nm}$ which belongs to the silver and platinum nanoparticles, respectively. The graphene-based bio-composites, i.e., GO-Ag-S3, GO-Ag-S4, and GO-Pt-P3 showed optimal \%LE of 88, 92, and 89\%, respectively. Whereas, CHI-Ag-S4, CHI-Pt-P3, and $\mathrm{CHI}$-Pt-P4 bio-composites showed optimal \%LE of 94,86 , and $94 \%$, respectively. Two regions, i.e., (1) between $3600-3100 \mathrm{~cm}^{-1}$, and (2) between 1,800 and $1,000 \mathrm{~cm}^{-1}$ in the FTIR spectra were found of particular interest. The FTIR profile exposed the available functional moieties at the surface of respective bio-composites. Variable mechanical attributes of silver and platinum nanoparticles loaded bio-composites were recorded from the stress-strain curves. All developed bio-composites showed bactericidal activities up to certain extent against both test strains. As compared to the initial bacterial cell count (control value, i.e., $1.5 \times 10^{8} \mathrm{CFU} / \mathrm{mL}$ ), the bio-composites with higher \%LE showed almost complete inhibition, with a $\log$ reduction from 5 to 0 , and bactericidal activities up to certain extent against both test strains, i.e., Bacillus subtilis (B. subtilis), and Escherichia coli (E. coli). In conclusion, the notable structural, functional, mechanical and antimicrobial attributes suggest the biomedical potentialities of newly in-house engineered silver and platinum nanoparticles loaded graphene and chitosan-based bio-composites.

Keywords: Nanoparticles, Bio-composites, Functional attributes, Characterization, Loading efficiency, Mechanical properties, Bactericidal, Biomedical

\section{INTRODUCTION}

An array of multi-functional biomaterials is evolving with enormous curiosity for researchers due to their range of applications. Research is underway around the globe to engineer pristine or hybrid polymer-based bio-composites that plays a substantial role in a field of catalysis, enzymology, environmental, pharmaceutical, and biomedical applications $\mathrm{s}^{1-5}$. Bio-composites can be engineered using naturally occurring biopolymers either in pristine form or the combination of multi-materials. Multi-functional materials-based bio-composites and/or nano-cues/nano-constructs have now become a high requisite for new applications ${ }^{6}$.
Likewise, synthetic polymer-based bio-composites, nano-composites (based on pristine or hybrid biopolymers) also exhibit inherited or improved structural and multi-functional attributes, for example, biocompatibility, biodegradability, (re)generatability, renewability, recyclability, high and efficient functionality against various substrates, induced turn-over, and overall cost-effectiveness are of high interest for numerous applications. Individually or collectively, all those properties of bio-composites open new and interesting perspectives with notable incidences in the environmental, biomedical, and biotechnological sector of the modern-day world ${ }^{5,7-9}$. 
Nowadays, the great emphasis has been given to exploit novel bio-composites to improve the existing characteristics or impart new properties of applied interests. To effectively address this concern, numerous nanoparticles, such as silver nanoparticles, platinum nanoparticles, and so on, are novel candidates that can be effectively used to engineer functionalized or nanoparticledecorated bio-composites. Incorporation of some nanoparticles furnishes new or improve the existing properties of the sample, such as antimicrobial, water vapor permeability, UV protection, facilitating the bio-composite suitability for food packaging, biomedical, and therapeutic applications $s^{10,11}$. For instance, the introduction of blended silica nanostructured particles in polymer matrix composites improved the creep resistance owing to homogeneous nanoparticle distribution ${ }^{12}$.

A great deal of research has been given to the nanoparticles-loaded/decorated constructs with multi-functional attributes for potential applications in the bio- and non-bio sectors of the modern world. Aiming to strengthen further the surface functionality critics of pristine or engineered bio-composites, numerous measures have been developed and exploited, such as re-functionalization of engineered constructs via a surface coating, dipping and decorating with other bioactive entities, e.g., nanoparticles. Nevertheless, the presence and utilization of numerous solvents or surfactants, along with other harsh reaction conditions all confines the synthesis and development of nanoparticles or nanoparticles-loaded bio-composites ${ }^{10,11}$. Regardless of the limitations mentioned above, the idea of using biopolymers, such as chitosan, cellulose, or graphene to engineer nanoparticlesloaded bio-composites has provoked significant research interests ${ }^{13}$. Though a massive spectrum of biopolymers is available, however, chitosan, and graphene are very promising and have been broadly employed in the biotechnology sector at large and biomedical, in particular ${ }^{14,15}$.

In view of the changing dynamics of $21^{\text {st }}$ century bio-composite materials with medical potencies, the incorporation of biologically active and therapeutic cues/agents into/onto the pristine bio-composite materials to engineer antibacterial matrices has received limited attention so far.
Thus, keeping in mind the points mentioned above and critics, herein, we report the development and characterization of nanoparticles-loaded biocomposites with unique structural and functional attributes. For a said purpose, chitosan and graphene were used to engineer bio-composites, which were then functionalized by loading silver and platinum nanoparticles on the surface. Finally, the nanoparticles-loaded bio-composites were then fully characterize using various instrumental techniques.

\section{MATERIALS AND METHODS Reagents/consumables}

All consumables or reagents/chemicals used in this study were of analytical laboratorygrade with purity $>99 \%$ and utilized deprived of any purification unless otherwise stated. Chitosan (MW 100-300 kDa with 82\% degree of deacetylation), graphite powder (with a purity $>99.99 \%)$, hydrogen peroxide $\left(\mathrm{H}_{2} \mathrm{O}_{2}\right)$, sodium borohydride $\left(\mathrm{NaBH}_{4}\right)$, glutaraldehyde, silver nitrate $\left(\mathrm{AgNO}_{3}\right)$, chloroplatinic acid $\left(\mathrm{H}_{2} \mathrm{PtCl}_{6}\right)$, sodium nitrate $\left(\mathrm{NaNO}_{3}\right)$, sulfuric acid $\left(\mathrm{H}_{2} \mathrm{SO}_{4}\right)$, potassium permanganate $\left(\mathrm{KMnO}_{4}\right)$, and ethylene glycol were obtained from the local suppliers and distributors of Sigma-Aldrich.

\section{Synthesis of silver and platinum nanoparticles}

The silver nanoparticles were synthesized using $50 \mathrm{mM}$ silver nitrate solutions. The $\mathrm{AgNO}_{3}$ reduction into $\mathrm{Ag}^{+}$ions was followed by mixing silver nitrate solutions. The reduction reaction was performed under continuous stirring for 10 min on a magnetic stirrer, and the solution was incubated at $28 \pm 2^{\circ} \mathrm{C}$ for $2 \mathrm{~h}$. A significant change in color from light yellow to blackish-brown was recorded that further indicates the reaction termination. While, platinum nanoparticles were developed by the reduction of $\mathrm{H}_{2} \mathrm{PtCl}_{6}$ in solution with a stabilizing or capping agent, i.e., ethylene glycol/ $\mathrm{NaBH}_{4}$ to form colloidal nanoparticles. As $\mathrm{H}_{2} \mathrm{PtCl}_{6}$ reduced to neutral platinum metal $\left(\mathrm{Pt}^{0}\right)$, the reaction mixture becomes supersaturated, the $\mathrm{Pt}^{0}$ begins to precipitate in the form of nanoscale particles. Both silver and platinum nanoparticles containing reaction mixtures were subjected to the centrifugation at $4,000 \mathrm{~g}$ for $5 \mathrm{~min}$, to purify the silver and platinum nanoparticles. The obtained supernatants were oven-dried at $50^{\circ} \mathrm{C}$ and subjected to UV-Visible spectrophotometric 
analysis. The $\lambda_{\max }$ values were noted from $0 \mathrm{~h}$ to each hour until $6 \mathrm{~h}$ and then after every $6 \mathrm{~h}$ until $24 \mathrm{~h}$ by taking an aliquot from the same mother liquor. The resultant final nanoparticles were stored and used for further characterization and loading onto graphene and chitosan-based biocomposites.

\section{Synthesis of graphene oxide}

Prior to the development of biocomposites, as received graphite powder was used to synthesize graphene oxide. For a said purpose, a mixture of graphite powder $(4 \mathrm{~g})$ and $\mathrm{NaNO}_{3}(2 \mathrm{~g})$ was prepared using the 2:1 ratio, respectively, in the presence of concentrated $\mathrm{H}_{2} \mathrm{SO}_{4}$ $(200 \mathrm{~mL})$. The above mixture was placed in an ice bath to maintain the temperature and cooled the mixture down to $0 \pm 1^{\circ} \mathrm{C}$. After attaining the requisite temperature $\left(0 \pm 1^{\circ} \mathrm{C}\right)$, the mixture was supplemented with $\mathrm{KMnO}_{4}(10 \mathrm{~g})$ consecutively with small doses and considered as a reaction solution. The reaction solution was heated up to $35 \pm 3^{\circ} \mathrm{C}$ and continuously stirred for $2 \mathrm{~h}$. This was followed by adding sterilized water $(300 \mathrm{~mL})$ to finalize the oxidation process. The unreacted $\mathrm{KMnO}_{4}$ from the reaction mixture was eliminated by adding $\mathrm{H}_{2} \mathrm{O}_{2}(30 \mathrm{~mL})$. After 30 min continuous stirring, the reaction mixture was centrifuged at $1500 \mathrm{~g}$ for $30 \mathrm{~min}$. The obtained supernatant was poured off, and the solid material was placed in water and recentrifuged under the same conditions. Finally, brown-yellow graphene oxide was recovered, quantified $(5 \mathrm{mg} / \mathrm{mL})$, and stored in a sterilized plastic tube.

\section{Nanoparticles-loaded graphene-based bio- composites}

Herein, silver and platinum nanoparticles loaded graphene-based bio-composites were prepared using a microwave-assisted approach. Briefly, graphene oxide powder $(10 \mathrm{mg})$ was poured into the ethylene glycol $(100 \mathrm{~mL})$ and treated by ultrasonication at a supersonic power of $500 \mathrm{~W}$ for $5 \mathrm{~min}$. This was followed by the addition of $\mathrm{AgNO}_{3}$ into the graphene oxide - ethylene glycol solution, the presence of $\mathrm{NaBH}_{4}$ as an additional reducing agent, and heated to $80 \pm 5^{\circ} \mathrm{C}$. This new mixture was then subjected to the irradiation in a microwave oven at $700 \mathrm{~W}$ for $2 \mathrm{~min}$. The resultant mixture was centrifuged at $1500 \mathrm{~g}$ for $20 \mathrm{~min}$, and solids were washed and dried in a hot air oven at $80 \pm 5^{\circ} \mathrm{C}$ for $12 \mathrm{~h}$ to obtain silver nanoparticles loaded graphene-based bio-composites. Likewise, for the development of platinum loaded graphenebased bio-composites, $\mathrm{AgNO}_{3}$ was replaced with $\mathrm{H}_{2} \mathrm{PtCl}_{6}$ as a platinum precursor. Sample IDs of newly in-house engineered silver and platinum nanoparticles loaded graphene-based biocomposites are summarized in Table 1.

\section{Nanoparticles-loaded chitosan-based bio- composites}

Herein, silver and platinum nanoparticles loaded chitosan-based bio-composites were prepared using the "one-pot" synthesis approach. For a said purpose, chitosan solution $(0.5 \%, \mathrm{w} / \mathrm{v})$ was sequentially added dropwise in $5.0 \%(\mathrm{w} / \mathrm{v})$ acetic acid solution under continuous stirring conditions at $28 \pm 2^{\circ} \mathrm{C}$ for $1 \mathrm{~h}$. Aiming to cast the

Table 1. Sample IDs of newly in-house engineered silver and platinum nanoparticles loaded graphene-based bio-composites

\begin{tabular}{lcccc}
\hline Sample IDs & Nanoparticle & NPs (\%) & NPs precursor & Bio-composite IDs \\
\hline S1 & Silver & 1 & $\mathrm{AgNO}_{3}$ & GO-Ag-S1 \\
S2 & Silver & 2 & $\mathrm{AgNO}_{3}$ & GO-Ag-S2 \\
S3 & Silver & 3 & $\mathrm{AgNO}_{3}$ & GO-Ag-S3 \\
S4 & Silver & 4 & $\mathrm{AgNO}_{3}$ & GO-Ag-S4 \\
S5 & Silver & 5 & $\mathrm{AgNO}_{3}$ & GO-Ag-S5 \\
P1 & Platinum & 1 & ${\mathrm{H} 2 \mathrm{PtCl}_{6}}_{6}$ & GO-Pt-P1 \\
P2 & Platinum & 2 & ${\mathrm{H} 2 \mathrm{PtCl}_{6}}_{6}$ & GO-Pt-P2 \\
P3 & Platinum & 3 & ${\mathrm{H} 2 \mathrm{PtCl}_{6}}_{6}$ & GO-Pt-P3 \\
P4 & Platinum & 4 & ${\mathrm{H} 2 \mathrm{PtCl}_{6}}_{6}$ & GO-Pt-P4 \\
P5 & Platinum & 5 & ${\mathrm{H} 2 \mathrm{PtCl}_{6}}_{6}$ & GO-Pt-P5
\end{tabular}


chitosan-based bio-composite membrane, the above reaction mixture was poured into the prelabeled and sterilized Petri dishes, followed by incubation in a hot air oven at $60 \pm 5^{\circ} \mathrm{C}$ for $24 \mathrm{~h}$. At the end of the stipulated incubation period, the casted chitosan-based bio-composite membranes were recovered and washed twice with sterilized water to remove the excessive acetic acid from the surface. The loading of silver and platinum nanoparticles onto the newly developed chitosanbased bio-composite membranes was performed via surface dipping and incorporation technique. For a said purpose, a pre-weight (Wi) chitosanbased bio-composite was dipped in a freshly synthesized silver and platinum nanoparticles solution, each separately. The reaction was activated using $0.5 \%(\mathrm{w} / \mathrm{v})$ glutaraldehyde solution (newly prepared within a $50 \mathrm{mM} \mathrm{Na-malonate}$ buffer of $\mathrm{pH} 4.5$ ). A pre-optimized reaction period (60 $\mathrm{min}$ ) was used to incubate the surface dipped chitosan-based bio-composite in a hot air oven at $50 \pm 5^{\circ} \mathrm{C}$ until thoroughly dried, and final dry weight (Wf) was recorded. Both Wi and Wf were used to calculate the nanoparticles loading efficiency. Sample IDs of newly in-house engineered silver and platinum nanoparticles loaded chitosan-based bio-composites are summarized in Table 2 .

Equation (1) was used to calculate the percent loading efficiency (\%LE):

Loading efficiency $(\%)=\frac{w f-w i}{w i} \times 100$

Where $\mathrm{Wf}=$ final dry weight and $\mathrm{Wi}=$ initial weight (chitosan without NPs).

\section{Instrumental analysis of pristine and engineered bio-composites}

AShimadzu UV-Visible spectrophotometer was used to record the absorbance of freshly synthesized silver and platinum nanoparticles. A quartz cell ( $1.0 \mathrm{~cm}$ path length) was used to record the absorbance of $300 \mu \mathrm{L}$ of silver and platinum nanoparticles, each separately, at the wavelengths from 200 to $600 \mathrm{~nm}$. The $\lambda_{\text {max }}$ values were noted from $0 \mathrm{~h}$ to each hour until $6 \mathrm{~h}$ and then after every $6 \mathrm{~h}$ until $24 \mathrm{~h}$ by taking an aliquot from the same mother liquor.

Fourier-transform infrared spectroscopy (FTIR) was used to investigate the available functional groups on the bio-composite surface. FTIR spectra were recorded at a wavelength range of $4000-500 \mathrm{~cm}^{-1}$ with 64 scans at a resolution of $4.0 \mathrm{~cm}^{-1}$ using a Perkin-Elmer System 2000 FTIR spectrophotometer. The possible peak numbers were assigned accordingly.

Mechanical measurements, i.e., Young's modulus, tensile strength, and elongation at break attributes of pristine and nanoparticlesloaded bio-composites were performed using a Perkin-Elmer Dynamic Mechanical Analyzer (DMA) Q800. For all analysis, the load was set within a range of 1-6000 $\mathrm{mN}$ with a crosshead speed of $200 \mathrm{mN} \mathrm{min}{ }^{-1}$ at a constant tensile rate.

\section{Evaluation of antibacterial attributes}

The antibacterial attributes of newly engineered bio-composites were trialed using a conventional spread-plate method. For a said purpose, two bacterial strains, i.e., Bacillus subtilis (B. subtilis), and Escherichia coli (E. coli). Before culturing the bacterial strains, each test

Table 2. Sample IDs of newly in-house engineered silver and platinum nanoparticles loaded chitosan-based biocomposites

\begin{tabular}{|c|c|c|c|c|}
\hline Sample IDs & Nanoparticle & NPs (\%) & NPs precursor & Bio-composite IDs \\
\hline S1 & Silver & 1 & $\mathrm{AgNO}_{3}$ & $\mathrm{CHI}-\mathrm{Ag}-\mathrm{S} 1$ \\
\hline S2 & Silver & 2 & $\mathrm{AgNO}_{3}^{3}$ & $\mathrm{CHI}-\mathrm{Ag}-\mathrm{S} 2$ \\
\hline S3 & Silver & 3 & $\mathrm{AgNO}_{3}^{3}$ & $\mathrm{CHI}-\mathrm{Ag}-\mathrm{S} 3$ \\
\hline S4 & Silver & 4 & $\mathrm{AgNO}_{3}^{3}$ & $\mathrm{CHI}-\mathrm{Ag}-\mathrm{S} 4$ \\
\hline S5 & Silver & 5 & $\mathrm{AgNO}_{3}^{3}$ & $\mathrm{CHI}-\mathrm{Ag}-\mathrm{S} 5$ \\
\hline P1 & Platinum & 1 & $\mathrm{H} 2 \mathrm{PtCl}_{6}^{3}$ & CHI-Pt-P1 \\
\hline P2 & Platinum & 2 & $\mathrm{H} 2 \mathrm{PtCl}_{6}^{6}$ & CHI-Pt-P2 \\
\hline P3 & Platinum & 3 & $\mathrm{H}_{2} \mathrm{PtCl}_{6}^{6}$ & CHI-Pt-P3 \\
\hline P4 & Platinum & 4 & $\mathrm{H} 2 \mathrm{PtCl}_{6}^{6}$ & CHI-Pt-P4 \\
\hline P5 & Platinum & 5 & $\mathrm{H} 2 \mathrm{PtCl}_{6}^{6}$ & CHI-Pt-P5 \\
\hline
\end{tabular}


specimen was UV-sterilized followed by controlled inoculation of freshly overnight grown bacterial suspensions (containing $10^{5} \mathrm{CFU} / \mathrm{mL}$ ) on the surfaces of the test bio-composites. The bacterially inoculated bio-composites were incubated at $35^{\circ} \mathrm{C}$ for $24 \mathrm{~h}$. At the end of the incubation, the overnight grown bacterial cells from the control and test bio-composites were wash away twice using $50 \mathrm{~mL}$ phosphate buffer $(\mathrm{pH}, 7.0)$. The bacterial cell count in terms of $\mathrm{CFU} / \mathrm{mL}$ was determined by conventional spread-plate method. For each test sample, the $\mathrm{CFU} / \mathrm{mL}$ values were used to calculate the antibacterial efficacy of the test composites using Equation 2.

Logreduction=Log CFU Control-Log CFU Treated

Due to the intrinsic variability of the antibacterial test results, at least a 2 -log reduction was considered necessary to claim an antibacterial activity, as reported by Elegir et al. ${ }^{16}$

\section{RESULTS AND DISCUSSION}

Nanoparticles confirmation via UV-Visible spectrophotometric analysis

Fig. 1 represents a UV-Vis spectroscopic profile of in-house engineered silver nanoparticles at numerous intervals within 0 to $24 \mathrm{~h}$ range. An evident change in color from colorless to blackishbrown was observed which was proportional to the reaction time. This evident intensity of the color change was mainly because of the reduction of $\mathrm{Ag}^{+}$to $\mathrm{Ag}^{0}$ accompanied by the Surface Plasmon Resonance (SPR) phenomenon ${ }^{11,17}$. Upon UV-Vis scan from 250 to $600 \mathrm{~nm}$, a characteristic peak appeared at the $\lambda_{\text {max }} 420 \mathrm{~nm}$ belongs to the silver nanoparticles. This appearance of broad peak at $420 \mathrm{~nm}$ also demonstrate the surface plasma resonance of silver nanoparticles ${ }^{10}$.

The UV-Vis spectroscopic profile of platinum nanoparticles is shown in Fig. 2. $\mathrm{H}_{2} \mathrm{PtCl}_{6}$ was reduced by ethylene glycol/NaBH4 to $\mathrm{PtCl}_{4}{ }^{2-}$ and $\mathrm{Pt}^{0}$. Upon UV-Vis scan from 200 to $800 \mathrm{~nm}$, a characteristic peak appeared at the $\lambda_{\max } 266 \mathrm{~nm}$ belongs to the platinum nanoparticles. According to the available literature, it is known that platinum forms complexes such as $\mathrm{Pt}\left(\mathrm{NO}_{2}\right)_{6}{ }^{2-}$ with nitrite ions $^{18}$. The nitrite ions may ligand exchange with $\mathrm{PtCl}_{6}{ }^{2-}$ to form complexes, such as $\mathrm{Pt}\left(\mathrm{NO}_{2}\right)_{6}{ }^{2-}$, which has been reported to have strong absorption peaks. The absorbance in the range from 250 to $350 \mathrm{~nm}$ is indicative of the $\mathrm{Pt}\left(\mathrm{NO}_{2}\right)_{6}{ }^{2-}$ species ${ }^{19}$.

\section{Percent loading efficiency (\%LE)}

The percent loading efficiencies of silver and platinum nanoparticles into/onto the

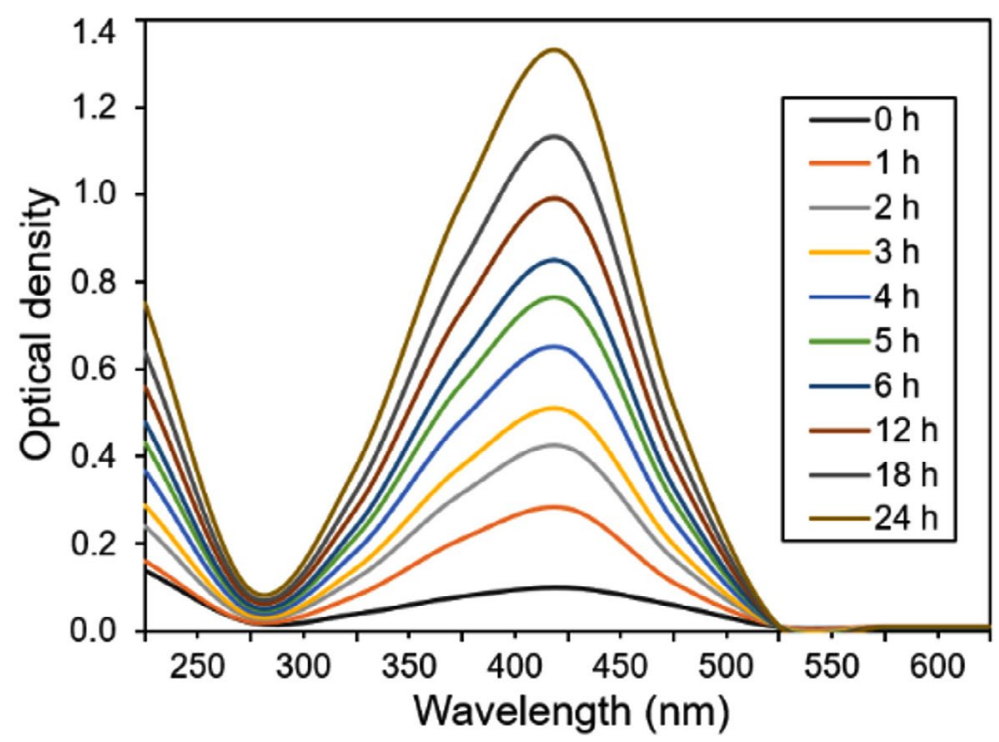

Fig. $1 \mathrm{UV}$-Vis spectral analysis of in-house engineered silver nanoparticles at numerous intervals within 0 to $24 \mathrm{~h}$ range. The $\lambda_{\max }$ values were noted from $0 \mathrm{~h}$ to each hour until $6 \mathrm{~h}$ and then after every $6 \mathrm{~h}$ until $24 \mathrm{~h}$ by taking an aliquot from the same mother liquor. 
graphene and chitosan-based bio-composites are shown in Fig. 3 and Fig. 4, respectively. For a precise record to observe the optimal \%LE, polynomial order 2 curve fit was inserted against each bio-composite. The threshold line was set at $80 \%$ value to distinguish and select the biocomposites with maximal percent LE values.
As evident in Fig. 3, the samples (S3, S4, and P3), i.e., GO-Ag-S3, GO-Ag-S4, and GO-Pt-P3 bio-composites showed optimal \%LE of 88, 92, and $89 \%$, respectively. While, rest of the biocomposites showed \%LE lower than the threshold set as per polynomial order 2 curve fit. As regards S1, S2, P1, and P2, the minimal \%LE could be due

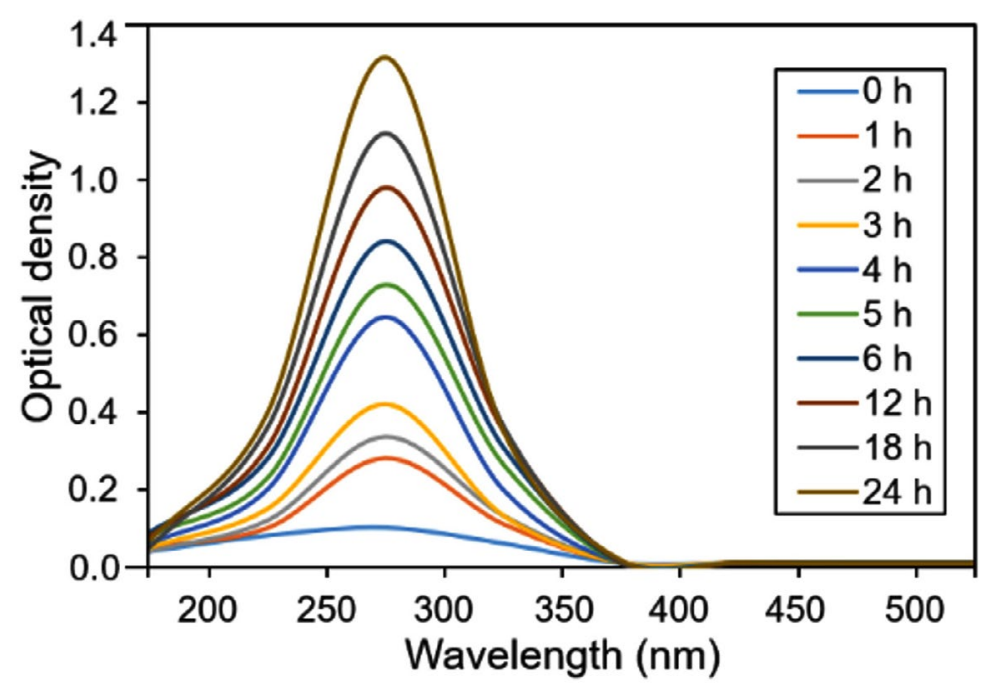

Fig. 2 UV-Vis spectral analysis of in-house engineered platinum nanoparticles at numerous intervals within 0 to 24 $\mathrm{h}$ range. The $\lambda_{\max }$ values were noted from $0 \mathrm{~h}$ to each hour until $6 \mathrm{~h}$ and then after every $6 \mathrm{~h}$ until $24 \mathrm{~h}$ by taking an aliquot from the same mother liquor.

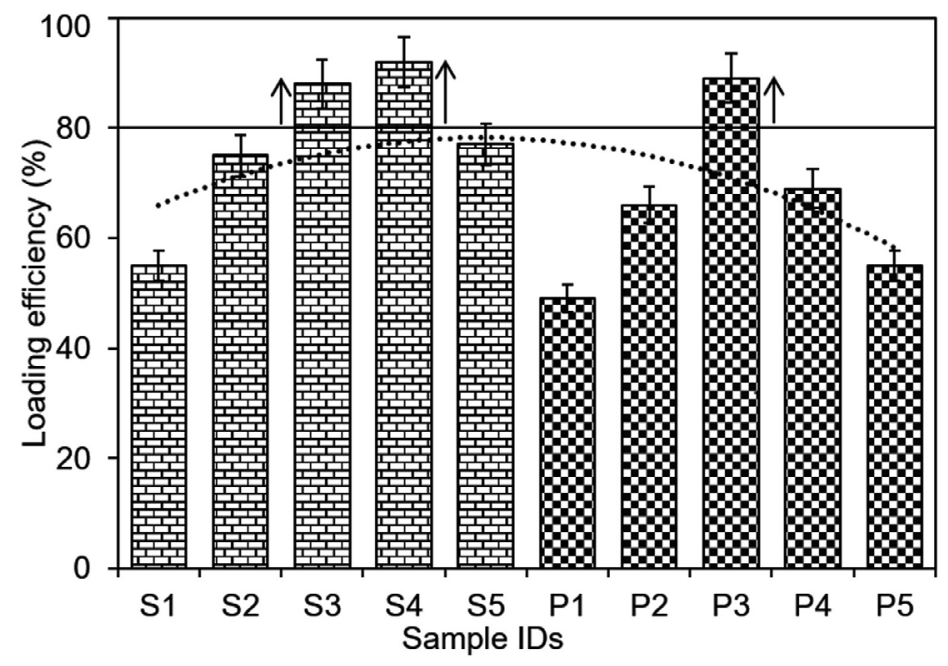

Fig. 3 Percent loading efficiency of newly in-house engineered silver and platinum nanoparticles into/onto graphenebased bio-composites. 
to the lower nanoparticles concentrations which were not good enough to reach the threshold set. Whereas, in case of S5, P4 and P5, though the loaded nanoparticles concentration was higher than S1, S2, P1, and P2, however, the higher concentration may have caused crowdedness and surface hindrance. This crowdedness and surface hindrance phenomenon may have limited an appropriate loading of nanoparticles and unloaded nanoparticles eroded away at the washing step. In case of chitosan-based bio- composites, as shown in Fig. 4, the samples (S4, P3 and P4), i.e., CHI-Ag-S4, CHI-Pt-P3, and CHI-Pt-P4 bio-composites showed optimal \%LE of 94, 86, and $94 \%$, respectively. All other bio-composites regardless of the lower and higher nanoparticles showed \%LE lower than the threshold set as per polynomial order 2 curve fit.

Fourier-transform infrared spectroscopy (FTIR)

The preliminary analysis of the newly engineered nanoparticles-loaded bio-composites was performed using Fourier-transform infrared

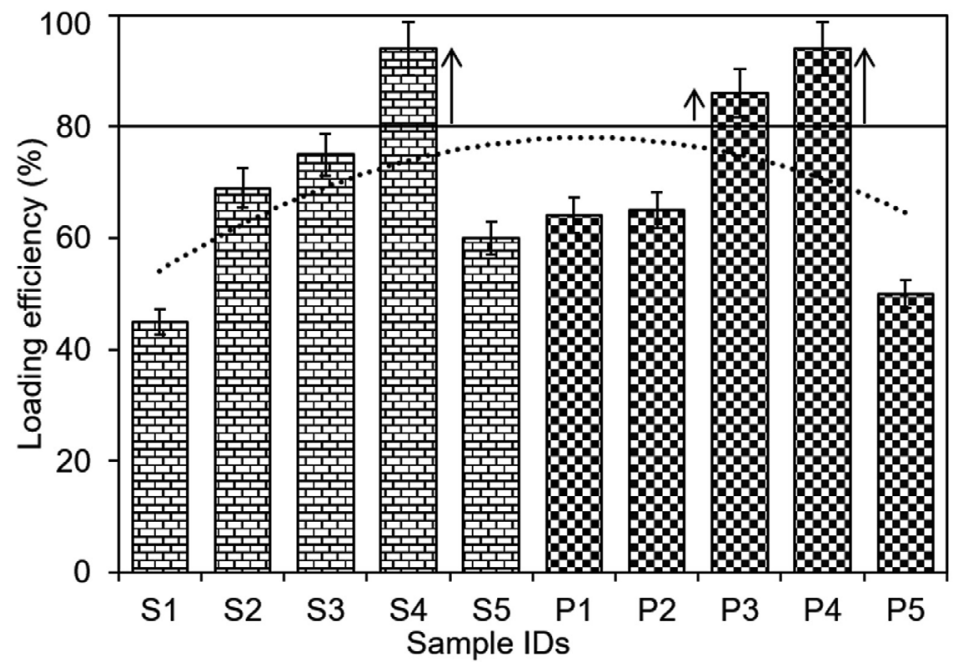

Fig. 4. Percent loading efficiency of newly in-house engineered silver and platinum nanoparticles into/onto chitosanbased bio-composites.

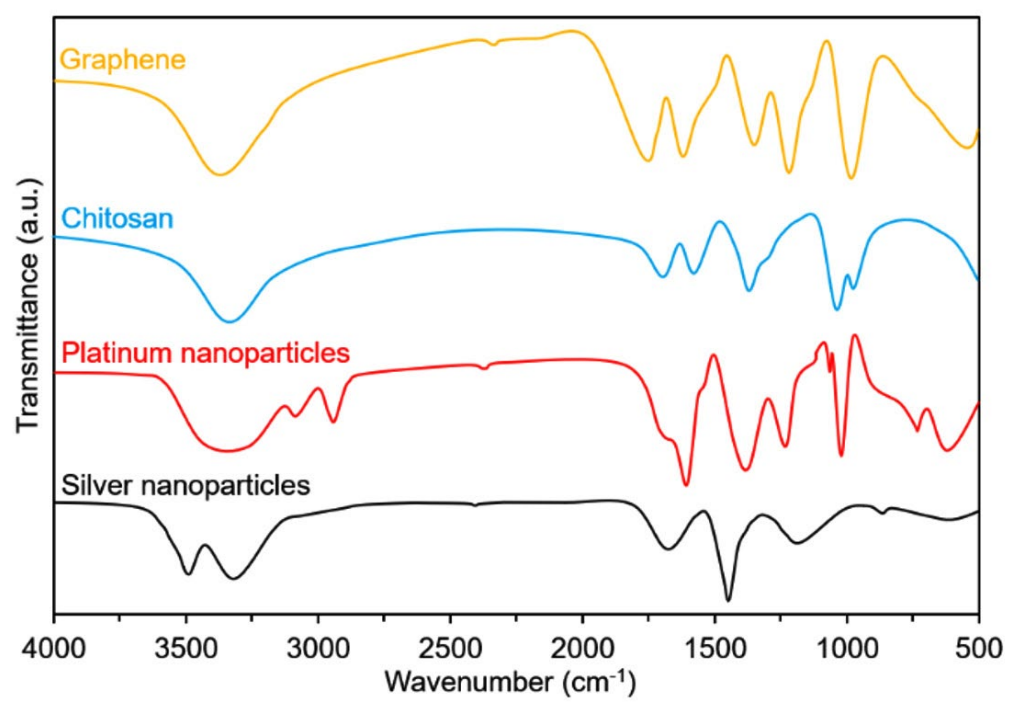

Fig. 5. FTIR profiles of respective polymeric materials and nanoparticles. 
spectroscopy (FTIR). Two regions, i.e., (1) between $3600-3100 \mathrm{~cm}^{-1}$, and (2) between 1,800 and 1,000 $\mathrm{cm}^{-1}$ in the FTIR spectra are of particular interest. The FTIR profiles, as shown in Fig. 5 (control), exposed the available functional moieties at the surface of respective polymeric materials and nanoparticles. Whereas, the typical FTIR profiles of silver and platinum nanoparticles loaded graphene-bio-composites are shown in Fig. 6 and Fig. 7, respectively. Fig. 8 and Fig. 9 illustrates FTIR profiles of silver and platinum nanoparticles loaded chitosan-bio-composites, respectively.
The characteristic peaks at 1115 and $1388 \mathrm{~cm}^{-1}$ correspond to $\mathrm{C}-\mathrm{O}-\mathrm{H}$ stretching and $\mathrm{C}-\mathrm{H}$ bending, respectively. The vibrational stretching of the $\mathrm{C}-\mathrm{C}$ is evident at $1588 \mathrm{~cm}^{-1}{ }^{20}$. The appearance of a broader peak at $3320 \mathrm{~cm}^{-1}$ refers to the characteristics of hydroxyl functional groups. The advent peak at $1640 \mathrm{~cm}^{-1}$ denotes to the capping of silver nanoparticles ${ }^{21}$. A sharp characteristic peak around $1,300 \mathrm{~cm}^{-1}$ regions is attributed to the $-\mathrm{NO}_{3}$ absorbance at the silver nanoparticles surface ${ }^{22}$. The peaks at $2930 \mathrm{~cm}^{-1}$ and $2875 \mathrm{~cm}^{-1}$ are mainly attributed to the $\mathrm{C}-\mathrm{H}$ bond in $-\mathrm{CH}_{2}$, and

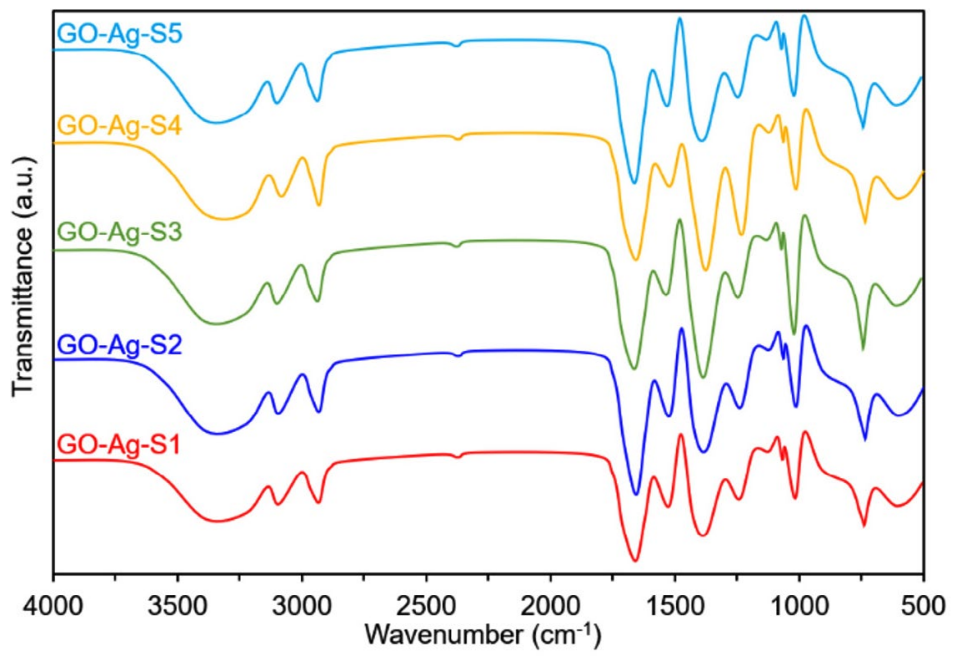

Fig. 6 Typical FTIR profiles of silver nanoparticles loaded graphene-bio-composites.

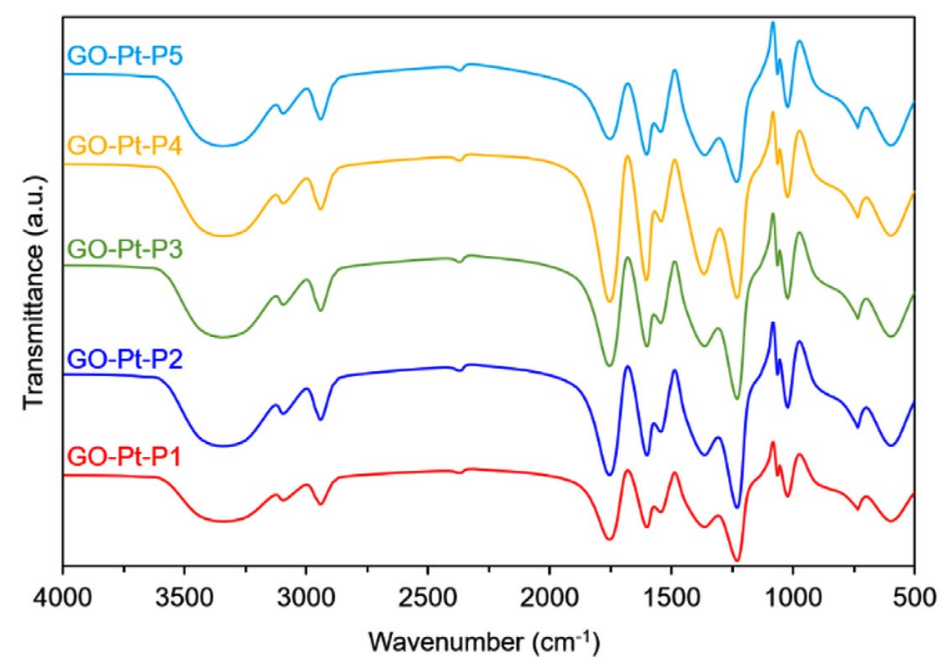

Fig. 7. Typical FTIR profiles of platinum nanoparticles loaded graphene-bio-composites. 
$-\mathrm{CH}_{3}$, respectively. From the characteristic range of $1680-1480 \mathrm{~cm}^{-1}$, the vibrational stretching of secondary amide, and amine groups was evident at $1660 \mathrm{~cm}^{-1}$ and $1580 \mathrm{~cm}^{-1}$, respectively. In case of graphene-based bio-composites, the existence of hydroxyl, carboxyl, and carbonyl functional entities was observed at $1413 \mathrm{~cm}^{-1}, 1728 \mathrm{~cm}^{-1}$, and 1050 $\mathrm{cm}^{-1}$, respectively ${ }^{23}$. The evident peak at $1600 \mathrm{~cm}^{-1}$ region is attributed to the $\mathrm{C}-\mathrm{C}$ vibrations from the graphitic domains. The appearance of a broad peak at $3260 \mathrm{~cm}^{-1}$ is due to the adsorbed water content in the surface of graphene.

\section{Dynamic Mechanical evaluation}

The mechanical attributes of the silver and platinum nanoparticles loaded graphene and chitosan-based bio-composites were recorded from DMA analysis. For a said purpose, the DMA tests were performed according to ASTM D-88297 standard test method. Table 3 provides the obtained results of mechanical profile of silver and platinum nanoparticles loaded graphene-based bio-composites. While the mechanical attributes of silver and platinum nanoparticles loaded chitosan-based bio-composites are given in Table 4. For both types of developed bio-composites, the mechanical attributes, i.e., tensile strength, young's modulus, and strain at break values were calculated from the stress-strain curves. Young's modulus, i.e., $E_{y}$, values of the bio-composites, i.e., GO-Ag-S1 and GO-Pt-P1 were recorded as 1050.20 MPa and 1072.30 MPa, respectively, which was decreased to $802.52 \mathrm{MPa}$ and 751.50 $\mathrm{MPa}$, as the silver and platinum nanoparticles loading concentration increased from $1 \%$ to

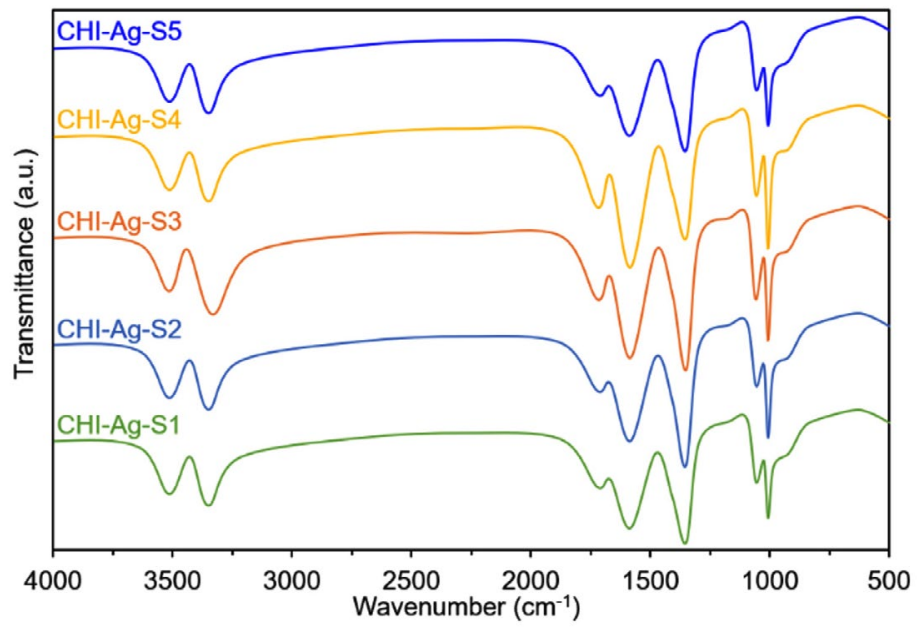

Fig. 8. Typical FTIR profiles of silver nanoparticles loaded chitosan-bio-composites.

Table 3. DMA-mechanical properties of silver and platinum nanoparticles loaded graphene-based bio-composites

\begin{tabular}{lccc}
\hline $\begin{array}{l}\text { Bio-composite } \\
\text { IDs }\end{array}$ & $\begin{array}{c}\text { Tensile } \\
\text { (MPa) strength }\end{array}$ & $\begin{array}{c}\text { Young's } \\
\text { modulus (MPa) }\end{array}$ & $\begin{array}{c}\text { Strain at } \\
\text { break (\%) }\end{array}$ \\
\hline GO-Ag-S1 & $35.50 \pm 3.33$ & $1050.20 \pm 123.50$ & $290 \pm 85.20$ \\
GO-Ag-S2 & $33.50 \pm 3.12$ & $1011.15 \pm 112.35$ & $266 \pm 76.35$ \\
GO-Ag-S3 & $31.45 \pm 2.14$ & $965.80 \pm 101.15$ & $230 \pm 52.20$ \\
GO-Ag-S4 & $27.25 \pm 2.19$ & $872.35 \pm 99.12$ & $165 \pm 32.55$ \\
GO-Ag-S5 & $25.80 \pm 3.10$ & $802.52 \pm 58.16$ & $151 \pm 35.70$ \\
GO-Pt-P1 & $36.75 \pm 3.80$ & $1072.30 \pm 133.33$ & $288 \pm 82.55$ \\
GO-Pt-P2 & $32.25 \pm 3.22$ & $1010.27 \pm 119.14$ & $259 \pm 71.45$ \\
GO-Pt-P3 & $26.31 \pm 2.88$ & $902.44 \pm 66.65$ & $202 \pm 69.66$ \\
GO-Pt-P4 & $27.45 \pm 2.12$ & $799.30 \pm 72.35$ & $188 \pm 45.45$ \\
GO-Pt-P5 & $22.35 \pm 1.16$ & $751.50 \pm 69.67$ & $162 \pm 31.35$ \\
\hline Journal of Pure and Applied Microbiology & \multicolumn{2}{c}{2332} &
\end{tabular}


$5 \%$. Likewise, the tensile strength and strain at break values showed the similar reduction pattern. As expected, this could be due to the presence of higher graphene part in GO-Ag-S1. Variable mechanical attributes of silver and platinum nanoparticles loaded graphene-based bio-composites were recorded from the stressstrain curves. Regardless of the nanoparticle type, i.e., either silver or platinum, a decreasing trend was observed in the tensile strength and percent elongation at break as the nanoparticle concentration increased. This could be related to two main factors: (1) random distribution of either silver or platinum nanoparticles in the chitosan membrane bio-composite, and (2) stress transfer through the nanoparticles-loaded bio-composite layers.

Antibacterial attributes of engineered biocomposites

Herein, a conventional spread-plate method was used to test the antibacterial attributes of silver and platinum nanoparticles loaded bio-composites against $B$. subtilis, and E. coli. The initial bacterial cell count, i.e., $1.5 \times 10^{8} \mathrm{CFU} / \mathrm{mL}$ was considered as a control value and used for comparison purposes for all test constructs. The results obtained against each bacterial strain for silver and platinum

Table 4. DMA-mechanical properties of silver and platinum nanoparticles loaded chitosan-based bio-composites

\begin{tabular}{lccc}
\hline $\begin{array}{l}\text { Bio-composite } \\
\text { IDs }\end{array}$ & $\begin{array}{c}\text { Tensile } \\
\text { (MPa) strength }\end{array}$ & $\begin{array}{c}\text { Young's } \\
\text { modulus (MPa) }\end{array}$ & $\begin{array}{c}\text { Strain at } \\
\text { break (\%) }\end{array}$ \\
\hline CHI-Ag-S1 & $22.35 \pm 1.11$ & $810.15 \pm 55.45$ & $86.50 \pm 5.23$ \\
CHI-Ag-S2 & $21.20 \pm 2.31$ & $830.32 \pm 49.56$ & $75.27 \pm 4.44$ \\
CHI-Ag-S3 & $17.62 \pm 1.15$ & $785.20 \pm 51.12$ & $63.45 \pm 3.76$ \\
CHI-Ag-S4 & $16.80 \pm 0.99$ & $640.65 \pm 35.55$ & $66.50 \pm 4.35$ \\
CHI-Ag-S5 & $13.35 \pm 1.53$ & $555.26 \pm 38.45$ & $53.50 \pm 3.33$ \\
CHI-Pt-P1 & $24.45 \pm 2.66$ & $765.58 \pm 44.43$ & $92.20 \pm 6.62$ \\
CHI-Pt-P2 & $21.32 \pm 1.16$ & $799.80 \pm 52.25$ & $81.20 \pm 6.15$ \\
CHI-Pt-P3 & $17.60 \pm 1.51$ & $610.10 \pm 42.28$ & $65.40 \pm 5.29$ \\
CHI-Pt-P4 & $18.32 \pm 1.88$ & $612.30 \pm 39.28$ & $58.55 \pm 4.36$ \\
CHI-Pt-P5 & $15.20 \pm 2.21$ & $576.48 \pm 30.15$ & $43.70 \pm 3.20$ \\
\hline
\end{tabular}

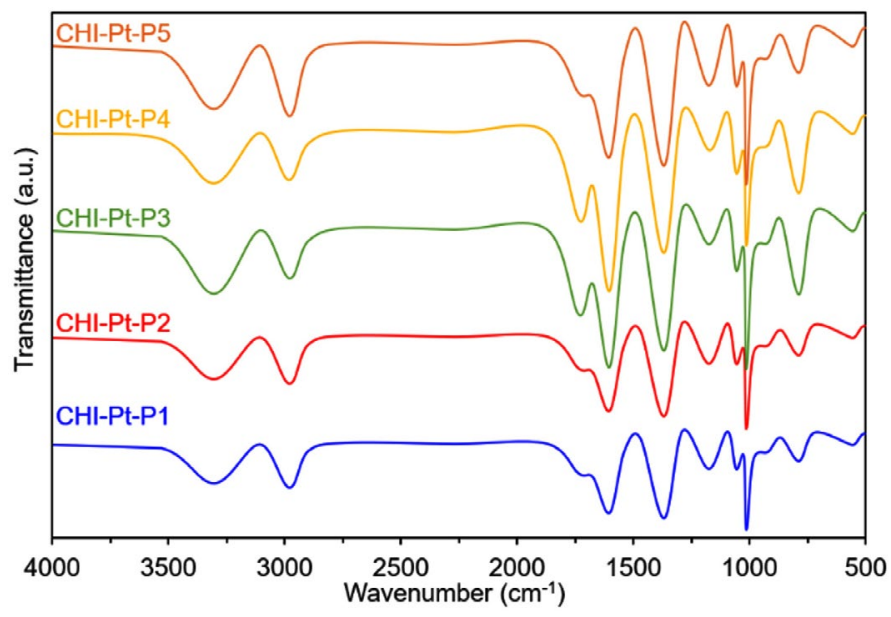

Fig. 9 Typical FTIR profiles of platinum nanoparticles loaded chitosan-bio-composites. 
nanoparticles loaded graphene, and chitosanbio-composites are shown in Fig. 10, and Fig. 11, respectively. Almost all developed bio-composites showed bactericidal activities up to certain extent against both test strains. More specifically, the bio-composites with higher \%LE showed almost complete inhibition with a log reduction from 5 to 0 . Comparatively, the graphene-based biocomposites were found slight less effective as compared to the chitosan-based bio-composites. The inherited antibacterial potential of chitosan may have also contributed synergistic effect along with the loaded nanoparticles. GO-Ag-S4 showed a complete bactericidal activity against B. subtilis. Whereas, GO-Ag-S3, and GO-Ag-S4 both bio-composites showed log reduction from 5 to 0.5 against $E$. coli. In case of platinum nanoparticle loaded graphene-based GO-Pt-P3 bio-composite, a maximal log reduction was from 5 to 0.5 against both test strains, i.e., B. subtilis, and $E$. coli. Likewise, CHI-Ag-S4 bio-composite showed log reduction from 5 to 0 against $B$. subtilis, and log reduction from 5 to 0.5 against $E$. coli. Whereas, CHI-Pt-P3, CHI-Pt-P4, and CHI-Pt-P5 bio-composites were found bactericidal as showed more than $2-\log$ reduction against both test
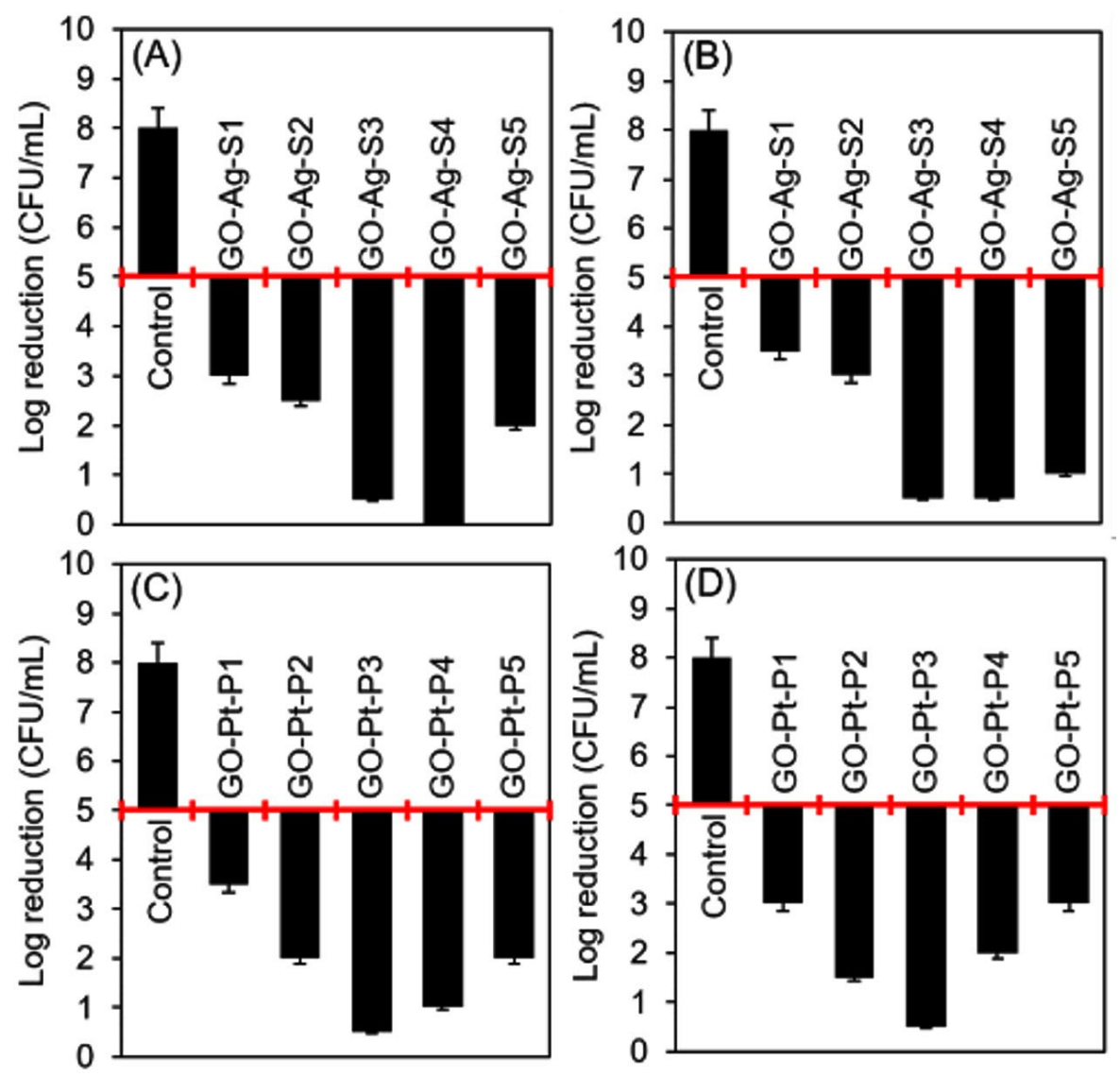

Fig. 10. Evaluation of the antibacterial activities of silver and platinum nanoparticles loaded graphene-biocomposites. $(A)$ and $(B)$ are representing the antibacterial attributes of silver nanoparticles loaded bio-composites against $B$. subtilis, and $E$. coli, respectively. (C) and (D) are representing the antibacterial attributes of platinum nanoparticles loaded bio-composites against $B$. subtilis, and $E$. coli, respectively. The reduction in the initial bacterial count i.e. $1.5 \times 10^{8} \mathrm{CFU} / \mathrm{mL}$ showed bacteriostatic and bactericidal activities of the respective samples. A 2-log reduction was considered to claim an antibacterial activity. 

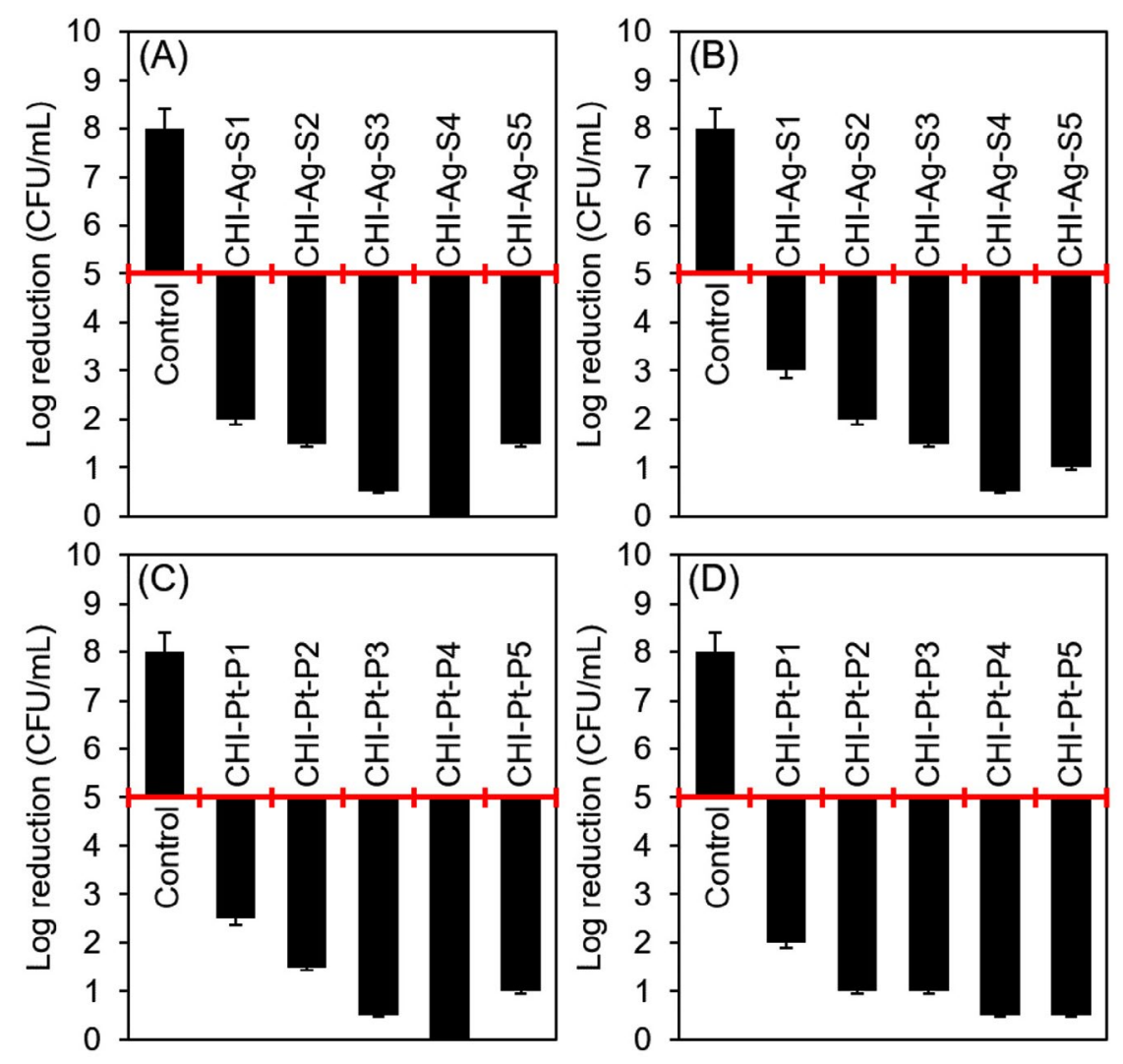

Fig. 11. Evaluation of the antibacterial activities of silver and platinum nanoparticles loaded chitosan-bio-composites. $(A)$ and $(B)$ are representing the antibacterial attributes of silver nanoparticles loaded bio-composites against $B$. subtilis, and E. coli, respectively. (C) and (D) are representing the antibacterial attributes of platinum nanoparticles loaded bio-composites against $B$. subtilis, and $E$. coli, respectively. The reduction in the initial bacterial count i.e. $1.5 \times 10^{8} \mathrm{CFU} / \mathrm{mL}$ showed bacteriostatic and bactericidal activities of the respective samples. A 2-log reduction was considered to claim an antibacterial activity.

strains, i.e., B. subtilis, and E. coli. The antibacterial mechanism of nanoparticles loaded constructs could be related to the membrane-disrupting, Reactive Oxygen Species (ROS), and DNA damage potentialities of AgNPs or AgNPs-based unique constructs $^{10,24}$.

\section{CONCLUSIONS}

In conclusion, the bio-composites developed using natural biopolymers/ biomaterials in combination with biologically active nanoparticles would prove to be robust in their preparation and performance. Herein, the newly in-house engineered silver and platinum nanoparticles loaded graphene, and chitosanbased bio-composites are anticipated to find applications in various biomedical sectors, where natural polymer-based bio-composites with unique structural and functional attributes are of utmost importance. As evident from the given results and characteristic profiles, the newly inhouse engineered bio-composites exhibited some unique functionalities and characteristics, such as multifunctional groups available at the surface, higher percent loading efficiency, good mechanical strength, and complete biocidal attributes.

\section{ACKNOWLEDGMENTS}

The authors would like to thank the FEMSA-Biotechnology Center at Tecnologico de Monterrey for providing technological and analytical services. The authors would also like 
to acknowledge the funding support provided by Tecnologico de Monterrey through the Bioprocess Research Chair (0020209113) and Biotechnology and Synthetic Biology Focus Group (002EICIP01). The financial support provided to Gustavo Hernandez-Vargas (CVU \# 634870) by the Consejo Nacional de Ciencia y Tecnologia (CONACYT) under the CONACYT National Scholarship program is thankfully acknowledged.

\section{CONFLICT OF INTEREST}

The authors declare that there is no conflict of interest.

\section{AUTHORS' CONTRIBUTION}

All authors listed have made a substantial, direct and intellectual contribution to the work, and approved it for publication.

\section{FUNDING}

The authors would like to recognize the funding support provided by Tecnologico de Monterrey through the Bioprocess Research Chair (0020209I13) and Biotechnology and Synthetic Biology Focus Group (002EICIP01).

\section{DATA AVAILABILITY}

All datasets generated or analyzed during this study are included in the manuscript and/or the Supplementary Files.

\section{ETHICS STATEMENT}

Not applicable.

\section{REFERENCES}

1. Shao W, Liu X, Min H, Dong G, Feng Q, Zuo S. Preparation, characterization, and antibacterial activity of silver nanoparticle-decorated graphene oxide nano-composite. ACS Applied Materials \& Interfaces. 2015;7(12):6966-6973. doi: 10.1021/acsami.5b00937

2. Essabir H, Raji M, Laaziz SA, Rodrique D, Bouhfid R. Thermo-mechanical performances of polypropylene biocomposites based on untreated, treated and compatibilized spent coffee grounds. Composites Part B: Engineering. 2018;149:1-11. doi: 10.1016/j. compositesb.2018.05.020

3. Bilal M, Nguyen TA, Iqbal HM. Multi-functional carbon nanotubes and their derived nano-constructs for enzyme immobilization-A paradigm shift in biocatalyst design. Coordination Chemistry Reviews. 2020a;422:213475. doi: 10.1016/j.ccr.2020.213475

4. Bilal M, Barcelo D, Iqbal HM. Nanostructured materials for harnessing the power of horseradish peroxidase for tailored environmental applications. Sci Total Environ. 2020b;749:142360. doi: 10.1016/j. scitotenv.2020.142360

5. Sosa-Hernandez JE, Villalba-Rodriguez AM, RomeroCastillo KD, et al. Poly-3-hydroxybutyrate-based constructs with novel characteristics for drug delivery and tissue engineering applications-A review. Polymer Engineering \& Science. 2020;60(8):1760-1772. doi: 10.1002/pen. 25470

6. Iqbal HM, Keshavarz T. Biopolyesters: Novel Candidates to Develop Multi-functional Biocomposites. In Biocomposites. Jenny Stanford Publishing. 2018:433455. doi: 10.1201/9781315110806-16

7. Iqbal HM, Kyazze G, Locke IC, Tron T, Keshavarz T. In situ development of self-defensive antibacterial biomaterials: Phenol-g-keratin-EC based biocomposites with characteristics for biomedical applications. Green Chemistry. 2015a;17(7):38583869. doi: 10.1039/C5GC00715A

8. Iqbal HM, Kyazze G, Locke IC, Tron T, Keshavarz T. Poly (3-hydroxybutyrate)-ethyl cellulose based bio-composites with novel characteristics for infection free wound healing application. Int J Biol Macromol. 2015b;81:552-559. doi: 10.1016/j. ijbiomac.2015.08.040

9. Iqbal HM, Keshavarz T. The challenge of biocompatibility evaluation of biocomposites. In Biomedical Composites. Woodhead Publishing. 2017:303-334. doi: 10.1016/B978-0-08-100752-5.00014-7

10. Bilal M, Rasheed T, Iqbal HM, Li C, Hu H, Zhang X. Development of silver nanoparticles loaded chitosanalginate constructs with biomedical potentialities. Int J Biol Macromol. 2017;105:393-400. doi: 10.1016/j. ijbiomac.2017.07.047

11. Bilal $M$, Zhao $Y$, Rasheed $T$, Ahmed I, Hassan ST, Nawaz MZ, Iqbal H. Biogenic nanoparticlechitosan conjugates with antimicrobial, antibiofilm, and anticancer potentialities: Development and characterization. Int J Environ Res Public Health. 2019;16(4):598. doi: 10.3390/ijerph16040598

12. Hassanzadeh-Aghdam MK, Ansari R, Mahmoodi MJ, Darvizeh A. Effect of nanoparticle aggregation on the creep behavior of polymer nano-composites. Composites Science and Technology. 2018;162:93-100. doi: 10.1016/j.compscitech.2018.04.025

13. Iqbal HMN, MV Rodriguez A, Khandia R, Munjal A, Dhama K. Recent trends in nanotechnology-based drugs and formulations for targeted therapeutic delivery. Recent Patents on Inflammation \& Allergy Drug Discovery. 2016 ;10(2):86-93. doi: 10.2174/187 2213X10666161213162823

14. Murata Y, Jinno D, Liu D, Isobe T, Kofuji K, Kawashima $\mathrm{S}$. The drug release profile from calcium-induced alginate gel beads coated with an alginate hydrolysate. Molecules. 2007;12(11):2559-2566. doi: 10.3390/12112559

15. Shi J, Alves NM, Mano JF. Chitosan coated alginate beads containing poly ( $\mathrm{N}$-isopropylacrylamide) for dual-stimuli-responsive drug release. Journal of Biomedical Materials Research Part B: Applied Biomaterials. 2008;84(2):595-603. doi: 10.1002/ jbm.b.30907 
16. Elegir G, Kindl A, Sadocco P, Orlandi M. Development of antimicrobial cellulose packaging through laccasemediated grafting of phenolic compounds.Enzyme Microb Technol. 2008;43(2):84-92. doi: 10.1016/j. enzmictec.2007.10.003

17. Mulvaney P. Surface plasmon spectroscopy of nanosized metal particles. Langmuir. 1996;12(3):788800. doi: 10.1021/la9502711

18. Chen J, Herricks T, Geissler M, Xia Y. Single-crystal nanowires of platinum can be synthesized by controlling the reaction rate of a polyol process. J Am Chem Soc. 2004;126(35):10854-10855. doi: 10.1021/ ja0468224

19. Herricks T, Chen J, Xia Y. Polyol synthesis of platinum nanoparticles: control of morphology with sodium nitrate. Nano Letters. 2004 ;4(12):2367-2371. doi: 10.1021/nl048570a

20. Chandran SP, Chaudhary M, Pasricha R, Ahmad A,
Sastry M. Synthesis of gold nanotriangles and silver nanoparticles using Aloevera plant extract. Biotechnol Prog. 2006;22(2):577-583. doi: 10.1021/bp0501423

21. Shankar SS, Ahmad A, Sastry M. Geranium leaf assisted biosynthesis of silver nanoparticles. Biotechnol Prog. 2003;19(6):1627-1631. doi: 10.1021/bp034070w

22. Janardhanan R, Karuppaiah M, Hebalkar N, Rao TN. Synthesis and surface chemistry of nano silver particles. Polyhedron. 2009;28(12):2522-2530. doi: 10.1016/j.poly.2009.05.038

23. Krishnamoorthy K, Veerapandian M, Zhang LH, Yun K, Kim SJ. Antibacterial efficiency of graphene nanosheets against pathogenic bacteria via lipid peroxidation. The Journal of Physical Chemistry C. 2012;116(32):1728017287. doi: 10.1021/jp3047054

24. Zhang XF, Liu ZG, Shen W, Gurunathan S. Silver nanoparticles: synthesis, characterization, properties, applications, and therapeutic approaches. Int J Mol Sci. 2016;17(9):1534. doi: 10.3390/ijms17091534 